\title{
La neuroética: ¿Un neologismo infundado o una nueva disciplina?
}

Andrea Slachevsky Ch., M D, PhD ${ }^{1}$

En la literatura científica y de divulgación ha aparecido recientemente un número no despreciable de neologismos neurológicos, tales como neuroeconomía, neuromarketing o neuroética. Ante la profusión de estos vocablos, es legítimo preguntarse si se trata sólo de términos a la moda, inventados de manera oportunista y sin mayor contenido, o si estos términos son realmente un intento honesto de definir nuevos campos en las neurociencias. No pretendemos en esta editorial comentar sobre la pertinencia de todos estos términos, sino que nos concentraremos en el concepto de neuroética y en la implicancia que esta disciplina tiene en el campo profesional de neurólogos y psiquiatras. Expondremos brevemente por qué esta disciplina es necesaria y hablaremos de dos aspectos de la neuroética particularmente interesantes para el desempeño diario de los especialistas clínicos de las enfermedades del sistema nervioso central.

\section{Nacimiento de la neuroética}

A diferencia de lo sucedido con la investigación en genética humana, hasta hace poco no existía un debate sobre la implicaciones éticas de las neurociencias ${ }^{1}$. Según Judy Illes, el término "neuroética" nace en 19892. En 2002, se realizó el primer simposio sobre neuroética "Neuroethics: M apping the Field" bajo el alero de la fundación Dana³. No existe en la actualidad una definición de consenso sobre esta disciplina. En un sentido amplio, la neuroética concierne las implicaciones sociales, legales y éticas de las neurociencias y los problemas éticos de la investigación en neurociencias ${ }^{4}$. Gazzaniga, en su libro Ethical Brain, propone una definición que constituye una buena síntesis del campo de la neuroética:"el análisis de cómo queremos enfrentar los aspectos sociales de la enfermedad, la normalidad, la mortalidad, el estilo de vida y la filosofía de vida informados por nuestra comprensión de los mecanismos cerebrales subyacentes". En resumen, "es -o debería ser- un esfuerzo por elaborar una filosofía de vida basada en el cerebro" ${ }^{\text {. }}$.

\section{La neuroética: ¿moda pasajera o necesidad fundamental?}

Las investigaciones actuales en neurociencias nos están permitiendo comprender, entre muchas cosas, las bases neurobiológicas de la conciencia, de los comportamientos sociales, de la moralidad, de la toma de decisiones y de las principales enfermedades psiquiátricas ${ }^{6-10}$.

1 Programa de Farmacología, Instituto de Ciencias Biomédicas y Departamento de Ciencias Neurológicas, Facultad de Medicina. Universidad de Chile. Unidad de Neurología Cognitiva y Demencias. Servicio de Neurología Hospital del Salvador.

Este trabajo fue financiado por el proyecto Fondecyt 1050175 
Gracias a estos avances, hoy es posible decir sin temor a equivocarse que el dualismo cartesiano cuerpo/mente carece de asidero científico. Cabe mencionar que el mismo Descartes, en su texto "I'Homme", que el filósofo prefirió no publicar por temor a la inquisición, escribió: "Los hombres estarán compuestos [... ] de un Alma y de un Cuerpo; y debo describirles primero el cuerpo separadamente, y después el alma separadamente; y, finalmente, mostrarles cómo estas dos $\mathrm{N}$ aturalezas deben ser juntadas y unidas para componer hombres que se nos asemejen" (citado por Changeux y Ricoeur (2006) pág 49) ${ }^{11}$. Aún más, al finalizar "L'H omme”, Descartes precisa "que no debe concebirse [... ] en esta M áquina [... ] ningún otro principio de movimiento y vida sino su sangre y espíritu agitados por el calor del fuego que arde continuamente, en su corazón, que no es de otra Naturaleza que la de todos los fuegos que están en los Cuerpos Inanimados" (citado por Changeux y Ricoeur (2006) pág 51) ${ }^{11}$.

La principal razón que explica la persistencia del dualismo cuerpo/mente es probablemente un problema de lenguaje, como señalan el filósofo Paul Ricoeur y el connotado neurocientífico Jean-Pierre Changeux en su famoso diálogo "Ce qui nous fair penser. La Nature et la Régle", cuando hablan de la existencia de un discurso de lo psíquico y de un discurso neuronal y de la necesidad de un tercer discurso, que forje y utilice un lenguaje común que ponga en correspondencia los objetos mentales del mundo interno, los objetos del mundo exterior y la actividad neuronal, es decir, un discurso que considere los elementos psicológicos como elementos físicos. Jean-Pierre Changeux, cuando afirma que "el pensamiento no puede pensarse sin el cerebro", nos ilustra cuan íntimamente imbricados están la mente y el cerebro ${ }^{11}$ (pág 66).

De este modo, las neurociencias nos permiten comprender los fundamentos de lo que somos, de nuestra esencia, y sus avances tienen importantes consecuencias sobre la manera en que nos pensamos como personas, agentes morales y seres espirituales ${ }^{1}$.

Por otro lado, los conocimientos que nos dan las neurociencias tienen consecuencias prácticas en diversos ámbitos que van más allá de esta disciplina. Los avances en los métodos de investigación en neuroimagen, por ejemplo, permiten monitorear el funcionamiento del cerebro, vulnerando la privacidad de la mente, y podrían permitir juzgar a una persona no sólo por sus acciones, sino también en función de sus pensamientos². De manera similar, los avances en neuropsicofarmacología pueden ser usados para manipular diferentes estados mentales del ser humano, tales como el estado de ánimo y ciertas habilidades cognitivas.

La nueva concepción del ser humano que las neurociencias favorecen y el poder que los conocimientos y técnicas de las neurociencias nos dan para examinar y modificar el comportamiento humano muestra claramente que el surgimiento de la neuroética como nueva disciplina constituye una necesidad fundamental.

Pero ¿por qué elegir un nuevo término para hablar de las relaciones entre neurociencia y ética? A mi entender, la repuesta a esta interrogante puede encontrarse en la siguiente reflexión de Amos $\mathrm{Oz}$ sobre la incorporación de nuevos vocablos o palabras extranjeras a las lenguas: "sabemos usted y yo que el secreto de la vitalidad de las lenguas vivas reside en su capacidad de asimilar prácticamente todas las palabras y conceptos con los que se encuentran" (pág 72) ${ }^{12}$.

\section{La problemática de la neuroética}

Los problemas de la neuroética pueden ser divididos en dos categorías:

- Los problemas relacionados con los avances técnicos de las neurociencias, tales como las implicancias del desarrollo de las neuroimagenes funcionales, de la psicofarmacología, de los implantes cerebrales y de la interfase cerebro-máquina. 
- Los problemas filosóficos derivados del entendimiento de las bases neurobiologías de la conciencia, la personalidad y la conducta.

A modo de ejemplo, me referiré muy brevemente a dos problemas de la neuroética que tienen particular importancia en nuestra profesión: i) el uso de fármacos para optimizar el funcionamiento cognitivo ("cognitive enhancers") en sujetos sanos sin lesiones cerebrales y ii) las implicancias de las neurociencias en la medicina legal.

En los últimos años, la farmacopea psicofarmacológica se ha incrementado con fármacos destinados al tratamiento de los trastornos cognitivos, en particular los trastornos atencionales y de memoria. Estudios en sujetos sanos han mostrado que estos tienen efectos en las capacidades cognitivas. Por ejemplo, el metilfenidato impacta positivamente sobre las capacidades atencionales y ejecutivas. Existe también un importante desarrollo de fármacos con el objetivo de mejorar las capacidades mnésicas. Si bien estos fármacos están destinados principalmente al tratamiento de sujetos con trastornos de memoria, es altamente probable que algunos de los productos en desarrollo incrementen las capacidades mnésicas de sujetos sanos. Por ejemplo, la amapakina, que potencia los mecanismos de LTP (Long Term Potentiation), mejora los rendimientos en tests de memoria de sujetos sanos. Según M arta Farah, el uso de estos fármacos en sujetos sanos conlleva tres problemas éticos fundamentales:

i. Razones de salud: desconocemos los efectos adversos de esos fármacos en sujetos sanos $y$, en particular, las consecuencias de su uso a largo plazo.

ii. Las consecuencias sociales de estas moléculas: son varias las preguntas que surgen ante la posibilidad de uso de esos fármacos: ¿Cómo puede el uso de estos fármacos afectar las relaciones sociales? ¿En qué situación quedarán los individuos que prefieren no consumir esos fármacos en relación con los que sí los consumen? Por ejemplo, la libertad de no consumirlos ciertamente puede estar amenazada en sociedades altamente competitivas, en las que se juzga a los sujetos primordialmente por su desempeño intelectual. Para comprender los alcances de esta interrogante baste recordar la polémica existente en el deporte de alta competición respecto del uso indebido de diferentes sustancias para mejorar el rendimiento de los deportistas. Adicionalmente, la disponibilidad de estos fármacos amplificará las inequidades sociales, puesto que las personas de altos ingresos podrán acceder más fácilmente a ellos que los de bajos ingresos.

iii. Razones de índole filosófica: El uso de estos fármacos cuestiona, entre otros, nuestros conceptos de esfuerzo personal, capacidad de superación, autonomía e incluso del valor de las personas comparado al valor de las cosas. ¿H acemos trampa si mejoramos nuestro rendimiento laboral con el uso de psicoestimulantes? ¿Estamos tratando a las personas como objetos si tratamos de mejorar con fármacos sus capacidades cognitivas o su personalidad?

Ciertamente, el debate sobre las consecuencias de los fármacos procognitivos sobrepasa el campo de nuestras especialidades, pero como prescriptores de estas sustancias y conocedores de sus efectos benéficos y adversos, es nuestro deber intervenir en este debate.

Por otro lado, los avances en la comprensión de las bases neurobiológicas de las conductas sociales, de la moralidad y toma de decisión tienen implicancias en nuestra concepción de la responsabilidad e imputabilidad. Recientemente, la Corte Suprema de Estados Unidos determinó que un adolescente podía ser condenado a muerte por un crimen, puesto que el estado de maduración cerebral de los adolescentes los hace plenamente responsable de sus actos $^{13}$. Diferentes estudios han mostrado que criminales y psicópatas presentan rendimientos menores a los de controles sanos en evaluaciones neuropsiquiátricas ${ }^{14,15}$. ¿Son, por lo tanto, los criminales imputables de sus actos? ¿Tendrán los avances en neurociencias algún 
impacto en nuestro sistema legal? En repuesta a estas interrogantes, Greene y Cohen sugieren que las neurociencias, al cambiar nuestra comprensión de la noción de libre albedrío y responsabilidad, tendrán probablemente un efecto transformador en las leyes. Más aún, según estos autores, es necesaria una interpretación alternativa de la responsabilidad no exclusivamente basada en el libre albedrío y el desarrollo de un sistema legal que no se limite a castigar los malos comportamientos, sino también que promueva los buenos comportamientos y proteja a los ciudadanos ${ }^{16}$.

El texto precedente tan sólo pretende ser una reflexión breve y probablemente sesgada sobre la neuroética. Es ante todo un intento de explicar por qué la neuroética es importante para SONEPSYN y por qué, en vista de los avances en neurociencias, debemos asumir un mayor rol público e intervenir en diversos debates nacionales en los cuales podemos contribuir como especialistas de las enfermedades del sistema nervioso.

\section{Referencias}

1. Farah M J. Neuroethics: the practical and the philosophical. Trends Cogn Sci 2005; 9 (1): 34-40.

2. Illes J. Neuroethics in a new era of neuroimaging. AJNR Am J N euroradiol 2003; 24 (9): 1739-41.

3. M arcus S J. Neuroethics: M apping the field. Conference proceedings. N ew York: The Dana Press (http://www.dana.org/neuroethics.cfm), 2002.

4. Illes J. Neuroethics. Defining the issues in theory, practice, and policiy. Oxford: Oxford University Press, 2006.

5. Gazzaniga M. The Ethical Brain. Chicago: Dana Press, 2005.

6. Bechara A, Van Der Linden M. Decision-making and impulse control after frontal lobe injuries. Curr Opin N eurol 2005; 18 (6): 734-9.

7. Dehaene $S, N$ accache $L$, Cohen $L$, et al. Cerebral mechanisms of word masking and unconscious repetition priming. Nat N eurosci 2001; 4 (7): 752-8.

8. Lieberman M D. Social Cognitive Neuroscience: A Review of Core Processes. Annu Rev Psychol 2006.

9. Moll J, de Oliveira-Souza R, Eslinger PJ: M orals and the human brain: a working model. N euroreport 2003; 14 (3): 299-305.

10. Orellana G, Slachevsky A, Silva J. M odelos neurocognitivos en la esquizofrenia: rol del córtex prefrontal Revista Chilena de Neuro-Psiquiatría 2006; 44 (1): 39-47.

11. Changeux J P, Ricoeur P. Ce qui nous fait penser. La nature et la règle. Paris: Editions O dile Jacob, 1998.

12. Oz A. Une histoire d'amour et de ténèbres. Paris: Gallimard, 2002.

13. Eastman N, Campbell C. N euroscience and legal determination of criminal responsibility. $\mathrm{Nat}$ Rev Neurosci 2006; 7(4): 311-8.

14. Blair K S, N ewman C, M itchell D G, et al. Differentiating among prefrontal substrates in psychopathy: neuropsychological test findings. N europsychology 2006; 20 (2): 153-65.

15. Frierson R L, Finkenbine R D. Psychiatric and neurological characteristics of murder defendants referred for pretrial evaluation. J Forensic Sci 2004; 49 (3): 604-9.

16. Greene J, Cohen J. For the law, neuroscience changes nothing and everything. Philos Trans R Soc Lond B Biol Sci 2004; 359 (1451): 1775-85.

Correspondencia:

Andrea Slachevsky Ch., M D, PhD

Independencia 1027. Santiago Chile

Fono: 56-2-9786050. Fax: 56-2-7372783

E-mail: aslachevsky@adsl.tie.cl 